Topiques, études satoriennes

Topoï Studies, Journal of the SATOR

\title{
Représentations du corps ivre dans la littérature et la peinture hispaniques
}

\section{Juan Ibeas and Lidia Vazquez}

Volume 5, 2021

Le manger et le boire dans la fiction narrative

URI: https://id.erudit.org/iderudit/1081521ar

DOI: https://doi.org/10.7202/1081521ar

See table of contents

Publisher(s)

SATOR, Société d'Analyse de la Topique Romanesque d'Ancien Régime

ISSN

2369-4831 (digital)

Explore this journal

Cite this article

Ibeas, J. \& Vazquez, L. (2021). Représentations du corps ivre dans la littérature et la peinture hispaniques. Topiques, études satoriennes / Topoï Studies, Journal of the SATOR, 5, 17-26. https://doi.org/10.7202/1081521ar

\section{Article abstract}

Wine has been linked to Spanish culture since the time of the Romans and early Christianity. In Spain, a land of vines since antiquity, the representation of the drunken body in literature and painting is an omnipresent topos since the Middle Ages. The pros and cons of this are found in medical, philosophical, moral and religious texts, but especially in novels, which warn against excess. This article studies how Spanish painters and writers developed the imaginary of wine in all its forms, be they masculine or feminine, solar or twilight, enthusiastic or drowsy, in their reflections, in their drawings and paintings, in poems or novels, in order to transmit the ideological transformation that was going to occur in Spain around this drink. For only the artist seems to preserve the tradition of the divine origin of wine, reclaiming its thaumaturgical role thanks to the Bacchic nectar. Spanish men and women suffer or benefit from the virtues of wine, the main alcoholic beverage in Spain until the 21st century.
This document is protected by copyright law. Use of the services of Érudit (including reproduction) is subject to its terms and conditions, which can be viewed online.

https://apropos.erudit.org/en/users/policy-on-use/ 


\section{Représentations du corps ivre dans la littérature et la peinture hispaniques}

Juan Ibeas, Lidia Vazquez

Universidad del Pais Vasco

Le vin est lié à la culture espagnole depuis le temps des Romains et de la première chrétienté. En Espagne, terre de vignes dès l'Antiquité, la représentation du corps ivre dans la littérature et la peinture est un $\operatorname{topos}^{1}$ omniprésent depuis le Moyen Âge. Le vin, nectar sacré, médical, festif, apparaît comme une liqueur vivifiante, mais non exempte de danger. Les «pour» et les « contre» parcourent textes médicaux, philosophiques, moraux, religieux mais surtout romanesques, qui mettent en garde contre l'excès. L'esprit s'élève mais s'égare, le corps devient énergique mais se transforme aussi en outre.

Le caractère positif du breuvage bachique dans l'histoire de l'Occident, lorsqu'il est ingurgité avec mesure, se voit altéré par la tradition judéo-arabe ibérique qui, depuis le $\mathrm{XII}^{\mathrm{e}}$ siècle, met en garde contre l'absorption du nectar biblique et annonce son rapprochement avec une certaine image anticléricale ${ }^{2}$. Est parvenu jusqu'à nous un texte juif du XII ${ }^{\mathrm{e}}$ siècle, de Joseph ben Meïr Zabara, Le Livre du plaisir, qui affirme : « Le vin aveugle les yeux, noircit les dents, efface la mémoire, et rend fou le sage [...] affaiblit le pouvoir du corps et paralyse ses membres dans leurs fonctions, et altère les nerfs qui les gouvernent $^{3} »$. Il existe aussi un manuscrit arabe, écrit en aljamiado, du $\mathrm{XIV}^{\mathrm{e}}$ siècle, qui, comme le texte précédent, condamne l'ingestion d'alcool :

Garde-toi de boire du vin, ni peu ni beaucoup, car il est l'ennemi de l'homme vil comme de l'homme honnête, il trompe tout le corps, même s'il est savant, parce que le vin fait faire grand mal, il fait perdre la loyauté à l'homme bon, provoque

\footnotetext{
${ }^{1}$ Le sens de topos ici est le sens général de "lieu commun".

${ }^{2}$ Selon la Bible, la Terre sainte produit du vin depuis des milliers d'années.

${ }^{3}$ Joseph ben Meïr Zabara, (Juif catalan de la fin du XII ${ }^{\mathrm{e}}$ siècle), trad. anglaise par Israel Abrahams, The Book of Delight, 1912, Philadelphia, The Jewish Publication Society of America. Sauf mention contraire, les traductions en français sont les nôtres.
} 
beaucoup de rixes et grande inimitié [...] et entraîne la mort et les blessures de beaucoup d'hommes ${ }^{4}$.

Ici, médecine et religion se donnent la main pour condamner les libations vinicoles, qui affaiblissent et le corps et l'âme. Mais la tradition chrétienne s'impose peu à peu et avec elle l'acceptation du vin comme aliment revigorant et, qui plus est, sacré. Le texte littéraire le plus ancien en castillan où apparaît la liqueur biblique est le Cantar de Mio Cid (vers 1200 : Le Poème du Cid). Mais c'est le poème signé par Lope de Moros au début du XIII ${ }^{\mathrm{e}}$ siècle, Razón de amor con los denuestos del agua y el vino ${ }^{5}$ qui en fait une vraie thématique. Chacun, de l'eau et du vin, chante ses vertus et relève les défauts de son adversaire. Le vin loue les prodiges qu'opère l'ivresse : « yo fago al çiego ueyer/ y al coxo corer/ y al mudo faublar/ y al enfermo organar ${ }^{6} »$.

Le premier grand personnage ivre de la littérature espagnole est, on ne saurait s'en étonner, un moine. Dans les Milagros de Nuestra Señora, Gonzalo de Berceo (vers 1246), nous parle de ce religieux, un régulier très dévot de la vierge :

Entró enna bodega un día por ventura,

Bebió mucho del vino, esto fo sin mesura,

Embebdose el locco, issio de su cordura,

Iogo hasta las viesperas sobre la tierra dura.

Bien a ora de visperas el sol bien enflaquido,

Recordó mala-mientre andaba estordido:

Issio contra la claustra hascas sin nul sentido:

Entendiengelo todos que bien avie bebido.

Peroque en sus piedes non se podie tener, Iba a la eglesia commo solia façer?

Tout à coup, le diable lui apparait, d'abord sous les traits d'un taureau furieux, puis d'un chien et enfin sous les apparences d'un lion féroce. Le corps aviné souffre les morsures,

\footnotetext{
${ }^{4}$ Texte arabe aljamiado du XIV ${ }^{\text {e }}$ siècle, publié par M. J. Müller dans les Sitzunsberichte der königlich Bayerischen Akademie der Wissenschaften, 1860, p. 201 et suiv. Notre trad. française de l'aljamiado.

${ }^{5}$ Raison d'amour avec la querelle de l'eau et du vin. Le poème, transcrit par son signataire, dont nous ne savons s'il est l'auteur ou le copiste, est écrit en une koinè avec prépondérance de castillan, hybride d'aragonais et mozarabe.

${ }^{6}$ M. Barra Jover, «Razón de amor », p. 123-53, v. 239-42. Le titre complet avec la querelle a été établi par Menéndez Pidal mais Barra Jover réduit le titre pour inclure le dialogue à l'intérieur avec un soustitre. « Je fais voir à l'aveugle / Et courir au boiteux/ Et parler au muet/ Et guérir au malade ».

${ }^{7}$ Strophes 463-65 : «Il pénétra dans la cave un jour par hasard, / Il but beaucoup de vin, sans aucune mesure ; / Le fou s'enivra, sortit de sa raison, / Demeura inerte sur la terre dure / Jusqu'à l'heure des vêpres. / Au moment des vêpres, le soleil déjà affaibli, / Il s'en souvint à peine, il marchait étourdi, / Il sortit vers le cloître presque sans le sens ; / Ils virent tous qu'il avait bu. / Même s'il ne tenait pas sur ses pieds, / Il alla vers l'église, comme à son habitude ». L'orthographe ancienne des citations en espagnol est maintenue. 
les coups de corne et de griffes, images poétiques du corps meurtri par l'excès de l'alcool. La vierge, en toréadora improvisée, sauve notre moine de ses visions, nées des vapeurs éthyliques.

Une variante du moine, l'ermite, à cause des libations excessives qui lui ont fait " perdre corps et âme » (v. 529), devient un criminel, un violeur et un assassin. Ainsi le trouvons-nous dans le Libro de Buen Amor. Dans ce beau texte, on retrouve l'ambivalence du vin :

Es el vino muy bueno en su mesma natura, muchas bondades tiene, si se toma con mesura, al que demás lo bebe, sácalo de cordura, toda maldat del mundo fase et toda locura.

Por ende fuye del vino, et fas buenos gestos quando fablares con dueñas, diles doneos apuestos ${ }^{8}$.

Son ingestion excessive, certes, rend fou, mais surtout anéantit le corps. Dans ces propos on trouve une part d'anticléricalisme, le vin étant traditionnellement associé au clergé :

Fase perder la vista, et acortar la vida, tira la fuerça toda, si s' toma sin medida, fase tembrar los miembros, todo seso olvida : a do es el mucho vino, toda cosa es perdida.

Fase oler el fuelgo, que es tacha muy mala, huele muy mal la boca, non ay cosa que l' vala, quema las asaduras, el fígado trascala : si amar quieres dueña, del vino bien te guarda.

Los omes embriagos ayna envegesçen, fasen muchas vilesas, todos los aborresçen, en su color non andan, sécanse et enmagresçen, a Dios lo yerran mucho, del mundo desfallesçen ${ }^{9}$.

\footnotetext{
${ }^{8}$ Juan Ruiz, Archiprêtre de Hita, Libro de Buen Amor (1330), 1974, p. 200-216. Strophes 548-49 : « De comment l'Amour apprend l'Archiprêtre à avoir de bonnes mœurs et surtout à se garder de boire beaucoup de vin blanc et rouge » (De cómo el Amor castiga al arçipreste que aya en sí buenas constunbres, é sobre todo que se guarde de bever mucho vino blanco é tynto). « Le vin est très bon dans sa nature même, / Il a beaucoup de vertus s'il est pris avec mesure ; / Celui qui en boit de trop, perd la raison ; / Il fait tous les maux du monde, et toutes les folies; / Donc, fuis le vin et fais de beaux gestes / Quand tu parleras aux femmes ».

${ }^{9}$ Strophes 544-46. «Il fait perdre la vue, et il abrège la vie, / Dérobe toutes les forces, s'il est pris sans mesure, / Il fait trembler les membres, il fait oublier toute raison : / Où il y a trop de vin, tout est perdu. / Il rend l'haleine nauséabonde, qui est un défaut très vilain, / La bouche pue, sans qu'on puisse y remédier, / Il brûle les entrailles, il trouble le foie : / Si tu veux aimer une dame, le vin ne te convient pas. / Les hommes ivrognes vieillissent vite, / Ils font maintes vilenies, ils sont de tous haïs, / Ils perdent la couleur, se dessèchent et noircissent. / Ils agissent souvent contre Dieu, et ils s'écartent du monde ».
}

Topiques, Études satoriennes, 5, 2020, Le Boire et le manger, https://journals.uvic.ca/index.php/sator/index 
La plus célèbre ivrogne de la littérature espagnole est, sans doute, la Celestina. Dans sa tragi-comédie Calixte et Mélibée (1499), Fernando de Rojas met en scène ce personnage féminin qui profère un des plus enthousiastes éloges des effets corporels et spirituels de la beuverie :

Pues de noche, en invierno, no hay tal escalentador de cama: que con dos jarrillos destos que beba cuando me quiero acostar, no siento frío en toda la noche. Desto aforro todos mis vestidos cuando viene la Navidad; esto me calienta la sangre; esto me sostiene continuo en un ser; esto me hace andar siempre alegre; esto me para fresca. Desto vea yo sobrado en casa que nunca temeré el mal año, que un cortezón de pan ratonado me basta para tres días. Esto quita la tristeza del corazón, más que el oro ni el coral; esto da esfuerzo al mozo e al viejo fuerza; pone color al descolorido, coraje al cobarde, al flojo diligencia; conforta los cerebros, saca el frío del estómago, quita el hedor del anhélito; hace potentes los fríos, hace sufrir los afanes de las labranzas; a los cansados segadores hace sudar toda agua mala, sana el romadizo e las muelas ; sostiénese sin heder en la mar, lo cual no hace el agua. Más propiedades te diría dello que todos tenéis cabellos. Así que no sé quien no se goce en mentarlo. No tiene sino una tacha: que lo bueno vale caro e lo malo hace daño. Así que con lo que sana el hígado, enferma la bolsa. Pero todavía con mi fatiga busco lo mejor, para eso poco que bebo. Una sola docena de veces a cada comida: no me harán pasar de allí, salvo si soy convidada como agora ${ }^{10}$.

Dans cet éloge paradoxal, la vieille entremetteuse, après avoir ressenti dans son propre corps les suites de l'ingestion vinicole, conseille aux jeunes et aux vieux de s'adonner à la boisson miraculeuse. La mesure, certes recommandée, est toutefois relative, et la conséquence d'un souci d'argent plutôt que de santé. À la réplique de Parmeno, qui argue

\footnotetext{
${ }^{10}$ Fernando de Rojas, Tragicomedia de Calixto y Melibea (1499), "IX ${ }^{\mathrm{e}}$ Acte". « Car la nuit, en hiver, rien ne réchauffe mieux le lit que lui : avec deux pichets [de vin] que je bois quand je veux me coucher, je n'ai plus froid de la nuit. Tous mes habits en sont remplis quand Noël arrive ; il m'allume le sang; il me soutient continuellement dans tout mon être; il me fait marcher joyeuse ; il me conserve fraîche. Tant que j'en verrai en abondance à la maison jamais je ne craindrai une mauvaise année, car alors un croûton de pain rongé me suffit pour trois jours. Il ôte la tristesse du cœur, plus que l'or et le corail ; il donne des forces au jeune et de la vigueur au vieux; il restitue la couleur à l'exsangue, le courage au lâche, la diligence à l'indolent ; il réconforte la cervelle, dégèle l'estomac, fait disparaître la mauvaise haleine ; il ressuscite les impuissants, il soulage les peines des labeurs; il fait transpirer les humeurs corrompues aux moissonneurs fatigués, calme la rage des dents ; il tient sans se gâcher la traversée de la mer, ce que l'eau ne fait pas. Je vous raconterais d'autres bienfaits mais vous êtes encore bien trop jeunes. En somme, je ne connais personne qui ne le vante pas. Il n'a qu'un défaut : que le bon vaut cher et le mauvais rend malade. Ainsi ce qui soigne le foie, fait mal à la bourse. Mais je m'efforce toujours de trouver le meilleur, pour le peu que je bois. Une seule douzaine de fois à chaque repas : on ne me fera pas boire davantage, sauf si je suis invitée comme c'est ici le cas ». Dans son Teatro Crítico Universal, le père Feijoo soutiendra également que le vin 'voyage' bien : Jerónimo Benito Feijoo, Teatro crítico universal, (1726-1740), Madrid, Joaquín Ibarra, rééd. 1775. t. I : 6-45, p. 159. «El vino que se transporta por altísimas montañas, se enfria mucho en ellas, y despues se calienta , tal vez demasiado, en los valles sin perder nada de su valor» («Le vin qui se transporte à travers de très hautes montagnes, y refroidit beaucoup, et après il chauffe peut-être trop dans les vallées, sans rien perdre de sa valeur $»)$.
}

Topiques, Études satoriennes, 5, 2020, Le Boire et le manger, https://journals.uvic.ca/index.php/sator/index 
que la sagesse populaire préconise trois verres, Célestine répond : «Hijos, estará corrupta

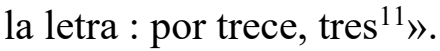

Si l'idée du vin comme pharmakon est historiquement avérée, au XVI siècle, la littérature picaresque se fait l'écho de ces pouvoirs curatifs et énergisants du vin. Dans le Lazarillo de Tormes (1554), l'auteur anonyme dit, par la voix de l'aveugle s'adressant au Lazarillo : «Lo que te enfermó te sana y da salud ${ }^{12}$ ». En effet, le Lazarillo, blessé par son maître avec le pichet de vin que le picaro vidait en cachette, va être soigné grâce à des ablutions avec cette même liqueur.

Dans le Quichotte, Cervantès métamorphose ses personnages en récipients à vin : Don Quichotte combat les outres comme s'il s'agissait d'une armée ennemie ; Sancho, dont le corps n'est pas sans rappeler la forme d'une gourde, tombe amoureux de sa bota : «[E]sta bota [...] es tan devota mía y quiérola tanto, que pocos ratos se pasan sin que la dé mil besos y mil abrazos ${ }^{13} »$. Quevedo (Romance XVII) aussi, dans un éloquent clin d'œil à la tradition carnavalesque de l'époque, transforme la peau de Roque, un des trois amis saouls d'une de ses romances, en cuir car son corps s'est changé en outre ${ }^{14}$.

Les rois d'Espagne, comme dans le reste de l'Europe, réfléchissent à la manière de réglementer la culture de la vigne, qui peut contribuer au développement économique du pays, tout en surveillant de plus en plus près les ivrognes. Charles V sera la première tête couronnée en Europe à émettre une ordonnance (7 octobre 1531) pour remédier aux beuveries et ivrogneries dans son Empire. François $\mathrm{I}^{\mathrm{er}}$ le suivra, en France, promulguant un édit (30 août 1536) qui fait de l'ivresse et de l'ivrognerie des crimes ${ }^{15}$. C'est, au fond, comme s'il existait deux phénomènes sans lien l'un à l'autre : la culture de la vigne et la production de vin réalisées par des hommes robustes et sains dans une Espagne tournée vers le progrès, enthousiaste, se réclamant de sa tradition bachique pour que la nation

\footnotetext{
${ }^{11}$ Fernando de Rojas, Ibid. « Mes enfants, il y a certainement une erreur dans le texte, c'est sans doute treize au lieu de trois ».

${ }^{12}$ Anonyme, Lazarillo de Tormes (1554). « Ce qui te rendit malade, te guérit et te redonne la santé ».

${ }^{13}$ Miguel de Cervantes, Don Quijote de la Mancha (1615), II Partie, chap. 51. « [L]a gourde est si dévote de ma personne et moi je l'aime tant qu'il ne se passe un moment sans que je l'embrasse et que je l'étreigne $»$.

${ }^{14}$ Romance 697, «Los Borrachos » (« Les ivrognes »), Madrid, 1648 v. 83-86.

15 «Et pour obvier aux oisivetez, blasphèmes, homicides et autres inconvéniens et dommages qui arrivent d'ébrieté : est ordonné, que quiconque sera trouvé yvre, soit incontinent constitué et détenu prisonnier au pain et à l'eau pour la première fois : et si secondement il est reprins, sera outre ce que devant, battu de verges ou de fouët par la prison : et la tierce fois sera fustigé publiquement; et s'il est incorrigible, sera puni d'amputation d'aureille, et d'infamie et banissement de sa personne » dans Isambert, Decrusy, Armet, Recueil général des anciennes lois françaises depuis l'an 420 jusqu'à la révolution de 1789, 1514-1746, t. XII, Paris, Chez Belin-Le-Prieur, p. 525.
}

Topiques, Études satoriennes, 5, 2020, Le Boire et le manger, https://journals.uvic.ca/index.php/sator/index 
espagnole rejoigne la France à la tête d'une Europe évoluée, riche et unie; et la consommation abusive des boissons alcoolisées qui conduit à la ruine des corps des ouvriers et des travailleurs des classes populaires en général. Car il est certain que l'imaginaire collectif de l'ivrogne connaît une évolution, glissant du personnage exultant et désinhibé de la fête populaire à l'individu déréglé de la ville. Ainsi, si la condamnation de l'ivrognerie à l'époque médiévale paraît reposer, d'une part, sur le fait qu'elle enfreint la mesure, règle d'or de la civilité de l'époque, et d'autre part, sur le fait qu'elle prédispose à d'autres péchés comme la colère, la luxure ${ }^{16}$ (même s'il est consommé avec modération, le vin échauffe le corps), à l'époque moderne, on va être dans un registre hygiéniste, dans l'idée que l'alcool compromet la santé du travailleur et par conséquent son rendement économique. De là à associer cette bête humaine, car l'homme se transformerait en brute sous les effets de l'alcool, aux crimes de la guerre, il n'y avait qu'un pas.

Nous retrouvons une matérialisation de cette évolution dans El triunfo de Baco (Le Triomphe de Bacchus) ${ }^{17}$ de Vélasquez (1629) : ce témoignage annonce dans cette période charnière la disparition de l'image mythique et l'apparition de la réalité sociale alarmante. Bacchus a certes tout d'un dieu dans cette toile qui l'éclaire pour mieux faire contraster sa peau divine avec les carnations violacées et rougeâtres de ses adorateurs. Mais à y regarder de près, le dieu aux airs caravaggiens est transformé en un être espiègle, aux lèvres charnues et sensuelles et au regard énigmatique, dirigé hors cadre, comme cherchant la complicité, sans se retourner, de son partenaire à gauche mais aussi, et surtout, du peintre lui-même qui lui a conféré sa rutilance, son énergie.

Ses compagnons, couronnés de feuilles de vigne comme lui, assistent amusés au couronnement du poète, seul être qui paraît encore prendre au sérieux le sacre bachique, et qui contraste avec les autres humains, véritable galerie physionomique d'ivrognes. Du plus jeune au plus vieux, du plus bouffi au plus incarnat, du sourire le plus niais aux yeux les plus injectés de sang et au nez le plus rouge, cet éventail d'hommes enivrés se regardant entre eux ou fixant le spectateur, sert de mise en garde contre ce peuple qui délaisserait tout en faveur de la beuverie collective. Le sens antique des bacchanales a disparu, nous avertit le maître. Mais on peut lire dans la toile que les hommes trouvent dans la sacralité du vin un soulagement à leurs souffrances.

\footnotetext{
${ }^{16}$ Voir La Queste del saint Graal.

${ }^{17}$ Musée du Prado : https://www.museodelprado.es/coleccion/obra-de-arte/los-borrachos-o-el-triunfo-debaco/4a23d5e2-9fd4-496b-806b-0f8ba913b3d8

Topiques, Études satoriennes, 5, 2020, Le Boire et le manger, https://journals.uvic.ca/index.php/sator/index
} 
Cette bacchanale mise à l'ordre du jour par Vélasquez se fait réaliste au siècle de la Raison, et le buveur quitte le décor idyllique pour la taverne, lieu de beuverie de groupe, espace de l'excès. Ainsi le reflète, dans un petit poème plein d'humour, José Iglesias de la Casa (1748-1791):
Batilo, échame vino,
Llena el vaso, muchacho:
Mira que no le llenas,
Échale hasta colmarlo.
Echa otra vez; pues este
Lo mismo que el pasado
De un sorbo le he bebido;
Con la misma sed me hallo.
Échame otra vez, que este
Le consumí de un trago:
Que o bien mi sed es mucha,
$\mathrm{O}$ me han mudado el vaso.
Otra vez echa, jay cosa! ${ }^{18}$

Au XVIII ${ }^{\mathrm{e}}$ siècle la Raison réunit en un seul discours, scientifique, philosophique et littéraire, la réprobation sociale de la démesure, l'inscrivant dans le topos $^{19}$ de l'abus de l'alcool au travail. Ce thème, caractéristique des Lumières, aura son écho le plus retentissant dans l'œuvre goyesque. Les hommes des Lumières espagnols vont appuyer les mesures des autorités espagnoles destinées à améliorer les conditions de travail et de vie du peuple, un peu à la manière des « Rues de la bière et du gin » de Hogarth ${ }^{20}$, qui soutiennent les nouvelles lois de Pitt en faveur de la consommation de la première, boisson nationale, salutaire, et contre l'abus du deuxième, alcool étranger, pernicieux.

En parallèle de ces figurations hogarthiennes, les corps souffrants des maçons, les religieux bouffis et titubants, les soldats chancelants et sans force, parcourent l'œuvre goyesque comme pour avertir des conséquences funestes de la consommation excessive d'alcool. El albañil herido (Le maçon blessé, huile sur toile, 1787) ${ }^{21}$ met en garde contre les suites néfastes de l'ingestion d'alcool au travail. Le corps de ce maçon, qui, ivre, a souffert d'un accident du travail, y apparaît délabré (il ne tient pas debout). Il est

\footnotetext{
${ }^{18}$ José Iglesias de la Casa, « Anacreóntica X », dans Poesías póstumas 1793-1798, 1869, p. 437. « Batilo, verse-moi du vin, / Remplis mon verre, mon garçon : / Regarde, tu ne le remplis pas, / Verses-en encore, jusqu'à ras bord. / Reverses-en encore, car celui-ci / Comme le précédent / D'un trait je l'ai bu ; / J'ai toujours la même soif. / Verses-en encore, car celui-ci / Je l'ai fini d'un coup. / Ou bien ma soif est grande / Ou bien on a changé mon verre. / Reverses-en encore, voyons ! »

${ }^{19}$ Le sens de "topos" ici correspond à celui de la SATOR.

${ }^{20}$ Tate Britain : https://www.tate.org.uk/tate-etc/issue-9-spring-2007/grandfather-satire et https://www.tate.org.uk/art/artworks/hogarth-gin-lane-t01799

21 Musée du Prado: https://www.museodelprado.es/coleccion/obra-de-arte/el-albail-herido/658a2cf1eb95-489c-b602-f3e3feda6b24
}

Topiques, Études satoriennes, 5, 2020, Le Boire et le manger, https://journals.uvic.ca/index.php/sator/index 
significatif de comparer ce tableau avec son dessin préparatoire (1786) : dans la toile définitive, destinée à être accrochée aux murs de la résidence royale, les traits des trois personnages sont sérieux, pathétiques, soulignant l'accident du travail et gommant la cause éthylique, qui saute aux yeux (il vient de vomir sur sa chemise, devant le regard amusé de ses compagnons, ivres comme lui) dans le dessin ${ }^{22}$.

L'autre grand topos des Lumières espagnoles sur l'ivresse sera celui de la dégénérescence du corps ivre. Alors que les physiocrates espagnols encouragent la culture de la vigne, le corps ivre apparaît comme malsain, donc pas rentable pour un marché du travail grandissant, et les ilustrados vont se servir de la fiction pour modérer la passion, pourtant si espagnole, pour le vin.

Mais, comme annoncé, l'artiste qui représente le mieux la laideur du corps ivre masculin est, sans aucun doute, Goya. Les corps de ses personnages sont boursoufflés, tels des outres à deux pattes, comme dans « Nadie nos ha visto », «Personne ne nous a vus » Caprice $79^{23}$, ou mutés dans une déformation monstrueuse, comme dans « Duendecitos », « Petits lutins », Caprice 4924, où des moines sont métamorphosés en lutins par les effet enivrants de la boisson, ou dans une animalisation dantesque, comme le vautour symbolisant la retraite d'une armée française dont les soldats sont connus pour leur goût démesuré du vin (Désastre 76) ${ }^{25}$.

Ces êtres saouls apparaissent, dans la plupart des cas, les traits défigurés par l'excès : les yeux qui louchent, les jambes écartées, le ventre enflé, les extrémités tétanisées (La era o el verano, L'aire ou l'eté, carton pour tapis, 1786-1787²6; « Buen sacerdote o dónde se ha celebrado », « Bon prêtre ou bien où a eu lieu la célébration », dessin préparatoire 1794-179727, du Caprice 18 : «Y se le quema la casa », « Et il laisse brûler sa maison», 1797-1798) ${ }^{28}$; les joues ballonnées («Soldado francés con un

\footnotetext{
${ }^{22}$ Goya aurait corrigé son idée première parce que la représentation des corps ivres paraissait choquer le roi, qui avait désiré voir soulignée sa réforme législative en faveur des conditions de travail des ouvriers, ce qui est mis en avant dans le tableau définitif.

23 Los Caprichos, Les Caprices, datent de1799. Fundación Goya en Aragón : https://fundaciongoyaenaragon.es/obra/nadie-nos-ha-visto/950

24 Musée du Prado : https://www.museodelprado.es/coleccion/obra-de-arte/duendecitos/d4c3825f-c96e42dc-af7f-62005899ff5e

${ }^{25}$ Los desastres de la guerra, Les désastres de la Guerre datent de 1814-1815. Musée du Prado: https://www.museodelprado.es/coleccion/obra-de-arte/el-buitre-carnivoro/2b99a9ce-3a6e-4951-b91c452e3506b7d6?searchid=ae456484-8f7d-8ccc-87f6-47ec84b1a352

${ }^{26}$ Musée du Prado : https:/www.museodelprado.es/coleccion/obra-de-arte/la-era-o-el-verano/ec1c94c1b21c-4330-9516-1c0d8078e87e

27 Fundación Goya en Aragón : https://fundaciongoyaenaragon.es/obra/buen-sacerdote-donde-se-hacelebrado/1026

28 Musée du Prado: https://www.museodelprado.es/coleccion/obra-de-arte/y-se-le-quema-lacasa/f71efdaf-5b2e-409a-9870-0f17da14c47b
}

Topiques, Études satoriennes, 5, 2020, Le Boire et le manger, https://journals.uvic.ca/index.php/sator/index 
acompañante borracho », "Soldat français avec un compagnon saoul», dessin de l'Album de Bordeaux, 1826) ${ }^{29}$, le regard hagard, la bouche ouverte, le rire niais, le corps à demi dénudé ; la démarche incertaine («El espartero borracho », « Le cordonnier saoul», dessin préparatoire 1796-1797) ${ }^{30}$. Cette dégénérescence du corps atteint également l'esprit. Ainsi Goya représente le pauvre ivrogne qui a perdu la tête ${ }^{31}$, détruisant une œuvre d'art classique (Dessin 1816-1820 : « No sabe lo que hace », « Il ne sait pas ce qu'il fait $»)^{32}$.

L'être humain, l'Espagnol, se voit ainsi, tour à tour, divinisé ou réifié, selon la qualité ou la quantité de la boisson ingurgitée, suivant le moment et l'espace, dépendant de la nature de l'acte plus ou moins socialisant, et bien sûr, le tout soumis aux aléas de l'âge, du sexe ou de la classe sociale. Le symbole christique du sang transformé en vin disparaît en faveur d'une approche profane et hygiéniste, et l'image de l'homme saoul tel un rieur qui rend comiques les situations dans lesquelles il intervient, topos scénique, se transforme progressivement en figure grotesque qui suscite une moquerie réprobatrice. Mais en général, c'est l'excès qui se voit condamné. Seul l'artiste paraît préserver la tradition de l'origine divine du vin, réclamant son rôle thaumaturgique grâce au nectar bachique. Hommes et femmes espagnols souffrent ou bénéficient ainsi des vertus du vin, la principale boisson alcoolisée en Espagne jusqu'au $\mathrm{XXI}^{\mathrm{e}}$ siècle.

\section{Bibliographie}

\section{Euvres et sources}

\footnotetext{
${ }^{29}$ Collection privée : https://www.elmundo.es/cultura/2015/07/08/559cdcbbe2704e52608b4574.html

${ }^{30}$ Musée du Prado : https://www.museodelprado.es/coleccion/obra-de-arte/el-espartero-borracho-que-noacierta-a-desnudarse/f79051f3-424c-4e37-877c-ebe08cddf22a

${ }^{31}$ Il s'agit d'un homme du peuple, payé comme d'autres par Ferdinand VII pour détruire les statues de la liberté. Il s'agit d'hommes recrutés dans les bas-fonds, qui s'enivrent pour mieux mener à bon terme l'ignoble tâche.

${ }^{32}$ Fundación Goya en Aragón : https://fundaciongoyaenaragon.es/obra/no-sabe-lo-que-hace/1139 
Anonyme, Lazarillo de Tormes (1554), version digitale de wikisource consultée le 20 septembre 2017 : https://es.wikisource.org/wiki/El_Lazarillo_de_Tormes.

BARRA JOVER, M., "Razón de amor". Texte critique et composition, dans Revista de Literatura Medieval, I (1989) ; p. 123-53, vv. 239-42.

CERVANTES, Miguel de, Don Quijote de la Mancha, IIe Partie, chap. 51. https://cvc.cervantes.es/literatura/clasicos/quijote/edicion/parte2/cap51/cap51_03. htm. Consulté le 10 août 2017.

FEIJOO, Jerónimo Benito, Teatro crítico universal, (1726-1740), Madrid, Joaquín Ibarra, rééd. 1775, t. I.

IGLESIAS DE LA CASA, José, « Anacreóntica X », Poesías póstumas 1793-1798, dans Leopoldo Augusto DE CUETO (ed.), Biblioteca de autores españoles, t. I, « Poetas líricos del siglo XVIII », Madrid, Rivadeneyra impr., 1869.

ISAMBERT, DECRUSY, JOURDAN, TAILLANDIER, Recueil général des anciennes lois françaises depuis l'an 420 jusqu'à la révolution de 1789, 1514-1746, t. XII, Paris, Chez Belin-Le-Prieur Paris, 1822-23.

MEÏR ZABARA, Joseph ben (Juif catalan de fin du XIIe siècle), trad. anglaise par Israel Abrahams, The Book of Delight, 1912 : www.gutenberg.org/ebooks/9886. Consulté pour la dernière fois le 13 septembre 2018.

ROJAS, Fernando de, Tragicomedia de Calixto y Melibea (1499), éd. digitale consultée le 20 septembre 2017: https://es.wikisource.org/wiki/La_Celestina.

RUIZ, Juan, Archiprêtre de Hita, Libro de Buen Amor (1330), Madrid, Espasa-Calpe, 1974 [J. Joset (ed.)].

MÜLLER, M. J., Sitzunsberichte der königlich Bayerischen Akademie der Wissenschaften, Munich, 1860 